\title{
Definition and Current Evaluation of Subfertile Men
}

\author{
Shai Shefi, Paul J. Turek \\ Departments of Urology and Obstetrics, Gynecology and Reproductive Sciences, University of \\ California San Francisco, San Francisco, California, USA
}

\begin{abstract}
Male infertility affects $10 \%$ of reproductive aged couples worldwide and is treatable in many cases. In addition to other well-described etiologies, genetic causes of male infertility are now more commonly diagnosed. Using principles of evidencebased medicine, this review outlines diagnostic and treatments options to inform clinical management. In order of importance, randomized controlled clinical trials, basic scientific studies, meta-analyses, case-controlled cohort studies, best-practice policy recommendations and reviews from peer-reviewed literature were incorporated that provide organized and timely guidelines to the current management of male infertility. The strength of evidence for treatment recommendations is also classified when appropriate.
\end{abstract}

Key words: male infertility; genetics; semen; spermatozoa; oligospermia; varicocele

Int Braz J Urol. 2006; 32: 385-97

\section{INTRODUCTION}

One in 6 couples trying to conceive will have difficulty. Infertility is defined as one year of unprotected intercourse without conception. On evaluation, roughly $50 \%$ of affected couples have causal or associated male factors as a cause of infertility. In addition, 1-10\% of male factor infertility is a result of an underlying, often treatable, but possibly life-threatening medical condition (1). For these reasons, the male evaluation is conducted systematically to acquire relevant information from the history, physical examination, semen analysis and hormone assessment. Current, evidence-based diagnostic and treatment algorithms for the management of male infertility are reviewed in this chapter. The strength of the evidence supporting a recommendation is graded according to Table- 1 .

\section{DIAGNOSIS}

\section{History}

The evaluation of male infertility should proceed in concert with the female as outlined in Figure-1. A thorough history is detailed in Table-2 and includes information about not only medical and surgical problems, but also developmental issues, occupational and social habits and exposures. Remember that sperm production is very sensitive to overall body health and problems that make the body ill will often affect spermatogenesis. The importance of information garnered from the male infertility history is derived from studies of male physiology as well as observational studies (Level C evidence).

\section{Physical Examination}

The physical examination assesses body habitus for obesity, gynecomastia and secondary sex 
Table 1 - Grades of evidence for management recommendations.

Grade of Evidence

Randomized, controlled clinical trials without heterogeneity

Randomized, controlled clinical trials with heterogeneity

Observational studies (cohort, case-controlled); expert consensus,

"proof of principle" studies

characteristics such as hair distribution. The phallus may reveal hypospadias, chordee, plaques or venereal lesions. The testes should be evaluated for their volume, consistency and contour irregularities suggestive of a mass. Since $80 \%$ of testis volume is determined by spermatogenesis, testis atrophy is likely

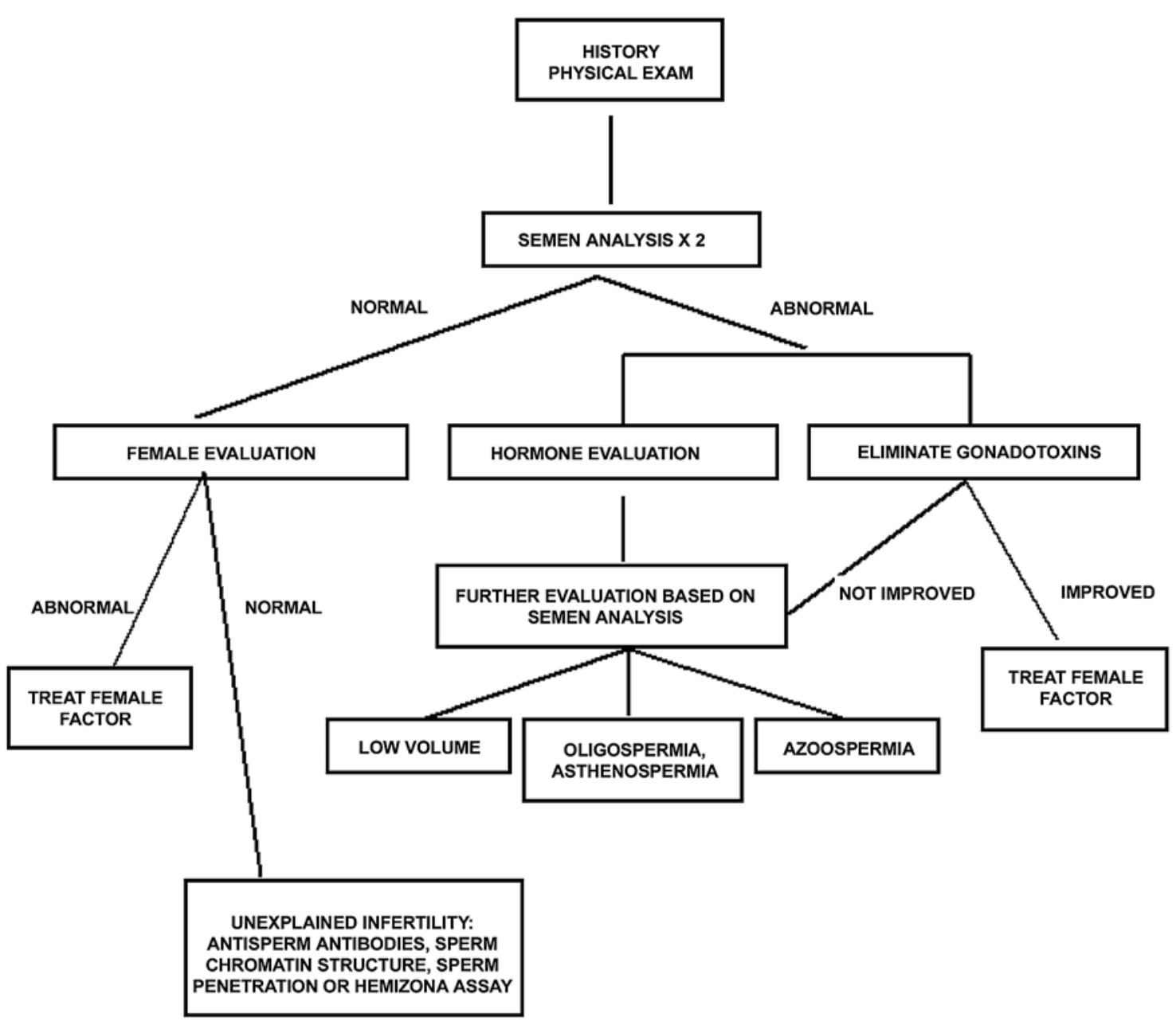

Figure 1 - Algorithm for diagnostic evaluation of male infertility. Adapted with permission from Turek PJ: Practical approach to the diagnosis and management of male infertility. Nature Clin Pract Urol. 2005; 2: 1-13. 
Table 2 - Components of the male infertility history.

\section{Medical History}

Fevers

Systemic illness-diabetes, cancer, infection

Genetic disease-cystic fibrosis, Klinefelter syndrome

\section{Surgical History}

Orchidopexy, cryptorchidism

Herniorraphy

Trauma, torsion

Pelvic, bladder, or retroperitoneal surgery

Transurethral resection for prostatism

Pubertal onset

\section{Fertility History}

Previous pregnancies (present and with other partners)

Duration of infertility

Previous infertility treatments

Female evaluation

\section{Sexual History}

Erections

Timing and frequency

Lubricants

Family History

Cryptorchidism

Midline defects (Kartagener syndrome)

Hypospadias

Exposure to diethylstilbestrol

Other rare syndromes-prune belly, etc.

\section{Medication History}

Nitrofurantoin

Cimetidine

Sulfasalazine

Spironolactone

Alpha blockers

Social History

Ethanol

Smoking/tobacco

Cocaine

Anabolic steroids

\section{Occupational History}

Exposure to ionizing radiation

Chronic heat exposure (saunas)

Aniline dyes

Pesticides

Heavy metals (lead) associated with decreased sperm production. Palpation of the epididymides might reveal induration, fullness or nodules indicative of infections or obstruction. Careful delineation of each vas deferens may reveal agenesis, atresia or injury. The spermatic cords should be examined for asymmetry suggestive of a lipoma or varicocele. Clinically significant varicoceles are diagnosed exclusively by physical examination. Lastly, a rectal examination is important in identifying large cysts, infections or dilated seminal vesicles.

\section{Semen Analysis}

Although not a true measure of fertility, the semen analysis, if abnormal, suggests that the probability of achieving fertility is lower than normal (2). Two semen analyses, performed with 2-3 days of sexual abstinence, are sought due to the large variability in semen parameters in healthy men (2). Lubricants should be avoided and the specimen processed during the first hour after ejaculation. There is recent debate concerning precisely which values are considered "normal". The World Health Organization currently recommends 20 million sperm/ $\mathrm{mL}$ and $50 \%$ motility as normal (2). However, a recent, controlled study of fertile and infertile couples suggested that a threshold of 48 million sperm $/ \mathrm{mL}$ and $63 \%$ motility best describes fertile semen (Grade B-C evidence) (3). Recent data also suggests that spermatogenesis takes $<60$ days to complete instead of 70-80 days as has been thought for 40 years, so that an individual semen analysis reflects biological influences occurring 2 months prior (4). The formal evaluation of sperm shape is termed morphologic assessment. Several descriptive systems exist to evaluate morphology. It is believed that sperm morphology may correlate with a man's fertility potential as reflected by in vitro fertilization (IVF) success (Level $\mathrm{C}$ evidence) (5). In general, the percentage of sperm with normal morphology has the greatest discriminatory power in distinguishing fertile from infertile semen, although no particular value is diagnostic of fertility or infertility (3). Sperm morphology complements the routine semen analysis in the male evaluation and better estimates the chances of fertility. 


\section{Hormonal Evaluation}

Current recommendations for endocrine evaluation of the infertile male are: a) sperm concentration < 10 million sperm $/ \mathrm{mL} ; \mathrm{b}$ ) erectile dysfunction; c) other clinical signs or symptoms suggestive of low testosterone or unrelated endocrinopathy. The initial evaluation should include serum testosterone and follicle stimulating hormone (FSH) levels. If the testosterone level is low, a repeat testosterone (total and possibly free testosterone) with luteinizing hormone (LH) and prolactin serum levels in a morning blood draw is advised. Although an endocrinopathy is found in $10 \%$ of tested men, clinically significant endocrinopathies are detected in $<2 \%$ of men (6).

\section{Genetic Evaluation}

Increasingly, genetic abnormalities are being identified as causes of male infertility. Thus, genetic testing should be performed in men with sperm concentrations $<10$ million sperm $/ \mathrm{mL}$, with detection of genetic anomalies increasing as sperm concentration decreases. Deletion of regions on the Y chromosome (microdeletions) occurs in $6 \%$ of men with severely low sperm counts and $13-15 \%$ of men with no sperm counts (7). Deletion of the DAZ (deleted in azoospermia) gene in the AZFc region is the most commonly observed microdeletion in infertile men. In addition, $2 \%$ of men with low counts and $15-20 \%$ of men with no sperm counts will harbor chromosomal abnormalities detected by cytogenetic analysis (karyotype). Patients at highest risk for abnormal cytogenetic findings include men with small, atrophic testes, elevated FSH levels, and azoospermia. These include conditions such as
Klinefelter syndrome and translocations of non-sex chromosomes. Table- 3 outlines current indications for genetic testing of infertile males (8).

Similarly, genetic testing is indicated for infertile men who present with cystic fibrosis $(\mathrm{CF})$ or the much more subtle condition, congenital absence of the vas deferens (CAVD). Similar genetic mutations are found in both patients, although CAVD patients are considered to have an atypical form of CF. Approximately $80 \%$ of men without palpable vasa will harbor a CF gene mutation. Recent data also suggest that azoospermic men with idiopathic obstruction and men with a clinical triad of chronic sinusitis, bronchiectasis, and obstructive azoospermia (Young syndrome) may be at higher risk for $\mathrm{CF}$ gene mutations.

\section{Other Testing}

\section{Anti-Sperm Antibodies (ASA)}

Testing for antisperm antibodies in indicated if: a) the semen analysis reveals aggregates of sperm; b) there is isolated asthenospermia; c) there is a risk of autoimmune infertility (i.e. prior torsion or testis injury); or d) there is unexplained infertility with a normal routine semen analysis. Usually performed with antibody coated, polyacrylamide spheres, an ASA test with at least $50 \%$ of sperm bound with antibodies is considered clinically significant. Occurring in 5-10\% of infertile men, it is thought that antibodies bound to the sperm head might interfere with sperm-egg interaction, penetration and fertilization, whereas tail bound antibodies may be more likely to affect sperm transport through the female reproductive tract (9).

Table 3 - Current indications for genetic testing of infertile men.

- A semen analysis with sperm concentrations $<10$ million sperm $/ \mathrm{mL}$ in a couple considering in vitro fertilization (IVF) and intracytoplasmic sperm injection (ICSI) (Y microdeletion assay and karyotype analysis).

- A semen analysis showing no sperm with testis atrophy in a couple considering testis sperm extraction with IVF and ICSI (Y microdeletion assay and karyotype analysis).

- A semen analysis shows no or low sperm concentration with at least one absent vas deferens on physical examination (cystic fibrosis gene mutations)

- A semen analysis showing no sperm with evidence of normal spermatogenesis (cystic fibrosis gene mutations)

- Syndromes or conditions suggested by personal or family histories (e.g. Kallman syndrome KAL 1-3) 


\section{Sperm Chromatin Structure Analysis}

Recent evidence suggests that the integrity of sperm DNA-chromatin packaging is important for male fertility. The structure of sperm chromatin (the DNAassociated proteins) can be measured by several methods, including the COMET and TUNNEL assays as well as by flow cytometry after acid treatment and staining of sperm with acridine orange (10). These tests assess the degree of DNA fragmentation that occurs after chemically stressing the sperm DNA-chromatin complex, and can indirectly reflect the quality of sperm DNA integrity. Abnormally fragmented sperm DNA rarely occurs in fertile men, but can be found in 5\% of infertile men with normal semen analyses and $25 \%$ of infertile men with abnormal semen analyses. This test can detect infertility that is missed on a conventional semen analysis. Often reversible, causes of DNA fragmentation include tobacco use, medical disease, hyperthermia, air pollution, infections, and varicocele. The current indication for this semen assessment is unexplained infertility.

\section{Post-Ejaculate Urinalysis (PEU)}

To diagnose retrograde ejaculation, the postejaculate urine should be inspected for sperm. A PEU can differentiate retrograde ejaculation from other causes of low ejaculate volume $(<1.5 \mathrm{~mL})$ including collection error, hypoandrogenism, ejaculatory duct obstruction, and congenital absence of the vas deferens.

\section{Semen Leukocyte Evaluation}

White blood cells (leukocytes) are present in all ejaculates and play important roles in immune surveillance and clearance of abnormal sperm. Leukocytospermia or pyospermia, an increase in leukocytes in the ejaculate, is defined as $>1$ million leukocytes $/ \mathrm{mL}$ semen and is a significant cause of male subfertility. The prevalence of pyospermia ranges from $3 \%$ to $23 \%$ of infertile men. In general, neutrophils predominate among inflammatory cells. This condition is detected by a variety of diagnostic assays, including differential stains (e.g., Papanicolaou), peroxidase stain that detects the peroxidase enzyme in neutrophils, and immunocytology. Such testing is indicated with infertility associated with elevated numbers of "round cells" in the ejaculate on routine semen analysis.

\section{Ultrasonography}

Renal ultrasonography is indicated to evaluate the possibility of unilateral renal agenesis in CAVD (10\% chance with bilateral vasal agenesis and $25 \%$ chance with unilateral vasal agenesis). Scrotal ultrasound is indicated to evaluate scrotal masses. It is controversial whether sonography should be used to detect subtle (subclinical) varicocele, since randomized trials of subclinical varicocele repair reveal no obvious benefit (Level A evidence). Transrectal ultrasonography (TRUS) is indicated for low semen volumes to exclude ejaculatory duct obstruction and to evaluate abnormalities on digital rectal examination.

\section{Vasography}

Formal imaging of the reproductive tract with vasography is warranted in cases of obstructive azoospermia. Vasography can be undertaken via scrotum, transrectal, transurethral or transperineal routes. Contrast material can delineate the proximal vas deferens, seminal vesicle, and ejaculatory duct anatomy, determine whether obstruction is present and delineate the anatomical site of obstruction. Sampling of vasal or seminal vesicle fluid during the procedure can also determine whether sperm exist within these structures, confirming obstruction and implying the lack of obstruction within the epididymis or testis. Vasography may also be indicated in the severely oligospermic patient when there is reason to suspect a unilateral obstruction (such as from a hernia repair) with an atrophic contralateral testis.

\section{TREATMENT OF MALE INFERTILITY}

The general treatment algorithm for male infertility is outlined in Figure-2. It is important to ensure that female reproductive potential is adequate to support the 6-12 month timeline associated with the treatment of most male factor issues. The decision to select classical treatments for male infertility including varicocele repair and vasectomy reversal rather than assisted reproduction is controversial, but accruing evidence from the urologic literature (Level 


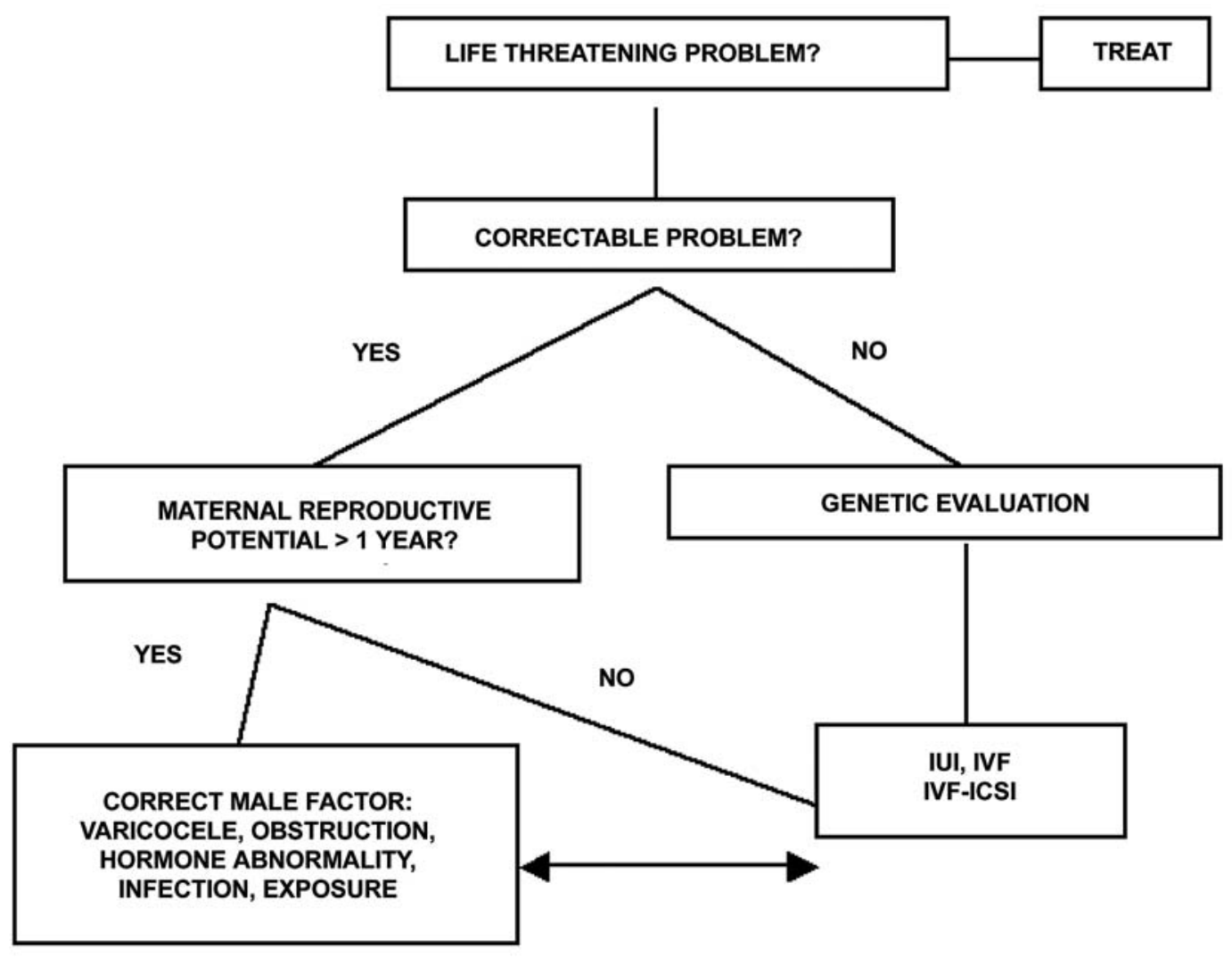

Figure 2 - Algorithm for treatment of male infertility. Adapted with permission from Turek PJ: Practical approach to the diagnosis and management of male infertility. Nature Clin Pract Urol. 2005; 2: 1-13.

B-C evidence) suggests that, in most male factor cases, correcting the male factor is cost-effective. This recommendation is supported by cost-benefit and decision modeling analyses $(11,12)$. Male factor infertility can be correctable or uncorrectable, and specifically treatable or not. These conditions are outlined below.

\section{Correctable Conditions in the Male}

\section{Coital Timing and Frequency}

Easily reviewed and corrected with counseling. An appropriate frequency for timed intercourse is every 2 days, performed within the periovulatory period with more emphasis on intercourse prior to, rather than following, ovulation (Level B evidence) (13). Home kits that detect the LH surge or the rising estradiol in the urine before ovulation are generally considered more reliable than basal body temperatures or untimed sexual intercourse. Coital toxins, including wet heat exposure from hot tubs or baths, cigarettes, cocaine, marijuana, and excessive alcohol should be avoided prior to, and during, intended conception.

\section{Abnormalities of Ejaculation}

Significant hypospadias can cause infertility due imprecise placement of the semen within the cervical os. Surgical correction or intrauterine 
insemination (IUI) of sperm are effective treatments. Severe phimosis, the inability to retract the foreskin, may lead to accumulation of sperm behind the preputial skin and result in a "low volume" ejaculate. To improve fertility and hygiene issues, gradual selfdilatation of the foreskin for cleaning or circumcision are recommended. Erectile dysfunction (ED) is frequently associated with infertility. Mostly psychogenic in nature, such cases are effectively treated with sexual counseling and phosphodiesterase inhibitors. For more severe ED due to organic causes, medical or surgical treatment is also effective. Retrograde ejaculation results from a failure of the bladder neck to close during ejaculation. It can be treated with a trial of sympathomimetic medications. Approximately $30 \%$ of men will respond to treatment; those most likely to benefit are patients with idiopathic causes or diabetes mellitus. The side effects of these medications usually limit the efficacy of this therapy. For medication failure, sperm harvesting techniques can be used with IUI to achieve a pregnancy.

\section{Medications}

Are usually tested extensively in animals for their potential as reproductive hazards before marketing. Despite this, it is wise to discontinue unnecessary medications that can be safely stopped during attempts to conceive. A list of gonadotoxic medications can be found in Table- 4 . These can result in infertility by various mechanisms. Ketoconazole, spironolactone, and alcohol inhibit testosterone synthesis, whereas cimetidine is an androgen antagonist. Recreational drugs such as marijuana, heroin, and opiates are associated with lower testosterone levels. Certain pesticides, like dibromochloropropane, are likely to have estrogenlike activity.

\section{Immunologic Infertility}

It is a complex problem. Several pathological conditions of the testis, including prior torsion, biopsy, injury or vasectomy, constitute risk factors for antisperm antibody (ASA) formation. Antibodies may disturb sperm transport or disrupt normal sperm-egg interaction. Antibodies may cause clumping or agglutination of sperm, which inhibits passage, or may block normal sperm binding to the oocyte. Available treatment options include corticosteroid suppression, sperm washing, intrauterine inseminations or IVF and ICSI. In general, if $>50 \%$ of sperm is bound with antibodies, then treatment should be offered. Treatment with corticosteroid immunosuppression should be considered for 6-9 months, and reliably lowers antisperm antibody levels but might not improve pregnancy rates (Level B evidence) (9). The risk of aseptic necrosis of the hip makes this treatment option relatively unattractive to most infertile couples. Intrauterine insemination places more sperm nearer

Table 4 - Drugs with potential adverse effects on male fertility.

- Alcohol
- Alkylating agents (e.g. cyclophosphamide)
- Allopurinol
- Antipsychotics
- Arsenic
- Aspirin (large doses)
- Caffeine
- Calcium channel blockers
- Cimetidine
- Cocaine
- Colchicine
- Dibromochloropropane (pesticides)
- Diethylstilbesterol (DES)

- Lead

- Lithium

- Monoamine oxidase inhibitors

- Marijuana

- Medoxyprogesterone

- Nicotine

- Nitrofurantoin

- Phenytoin

- Spironolactone

- Sulfasalazine

- Testosterone

- Tricyclic antidepressants

- Valproic acid 
the ovulated egg to optimize the sperm-egg environment and can be effective in cases of tailbound antisperm antibodies. IVF and ICSI very effectively bypass ASA-related infertility due to either poor sperm transport through the female reproductive tract, or poor sperm-egg interaction.

\section{Genital Tract Infection}

The agents most commonly responsible for male genital tract infections are listed in Table-5. Although genital tract infection has been linked to infertility in epidemiologic studies, the correlation between individual organisms and infertility is unclear. However, various products of activated leukocytes that coexist with genital infections may impair semen quality. A correlation exists between leukocytes in semen and the generation of superoxide anions, hydrogen peroxide, and hydroxyl radicals (reactive oxygen species), all of which can damage sperm membranes. Sperm are highly susceptible to the effects of oxidative stress because they possess little cytoplasm and therefore little antioxidant activity. Damage to sperm from oxidative stress has been correlated to loss of function and damaged DNA. Given that $83 \%$ of all infertile men will have positive semen cultures and that the relationship between bacterial cultures and infertility is at best inconclusive, semen cultures should be obtained only when there are features suggestive of infection, including: (1) a history of genital tract infection, (2) abnormal expressed prostatic secretion, (3) the presence of $>1000$ pathogenic bacteria/mL semen, and (4) the presence of $>1$ million leukocytes/mL semen (pyospermia). Uncontrolled studies suggest that pregnancy rates may improve after antibiotic treatment, but controlled studies do not confirm these findings. Antioxidant treatment may accompany antibiotics in cases of suspected infection.

\section{Hormonal Dysfunction}

Effective treatment of hormonal disorders usually involves reversing the specific abnormality detected. Examples of very correctable conditions include: hyperprolactinemia, hypothyroidism, congenital adrenal hyperplasia, and testosterone excess or deficiency due to steroids or genetic conditions like Kallman syndrome. These conditions should be sought and treated as suggested in Figure-2.

\section{Varicocele}

Defined as dilated and tortuous veins within the pampiniform plexus, the varicocele is the most common surgically correctable cause of male infertility. Varicocele is a disease of puberty, and is found in $15 \%$ of healthy young men, but in $40 \%$ of infertile men. In general, varicoceles do not spontaneously regress. The cornerstone of varicocele diagnosis rests on an accurate physical examination. The use of scrotal ultrasound to detect clinically unapparent (subclinical) varicoceles is currently unjustified given the lack of treatment efficacy demonstrated in 3 randomized, controlled trials (Level A evidence) (14). Precisely how varicoceles exert an effect on the testis remains unclear. Several theories have been postulated and it is likely that a combination of them results in infertility. These include: pituitarygonadal hormonal dysfunction, internal spermatic vein reflux of renal or adrenal metabolites, an increase in hydrostatic pressure with venous reflux, and an inhibition of spermatogenesis through the reflux of warm corporeal blood around the testis and elevation

Table 5 - The most common organisms in male genital infection.

\begin{tabular}{ll}
\hline - Neisseria gonorrhoeae & - Cytomegalovirus \\
- Chlamydia trachomatis & - Herpes simplex II \\
- Trichomonas vaginalis & - Human papilloma virus \\
- Ureaplasma urealyticum & - Epstein-Barr virus \\
- E. Coli (other gram neg. bacilli) & - Hepatitis B virus \\
- Mycoplasma hominis & - HIV
\end{tabular}


of intratesticular temperature. Regardless of the biological mechanism, there is strong evidence to suggest that varicoceles affect semen quality (15).

The finding of semen abnormalities constitutes the main indication for varicocele surgery in infertile men. Varicocele repair should also be considered in adolescents with a large varicocele and evidence of testis hypotrophy, and in the presence of varicocele-induced orchialgia. It is important to ensure adequate maternal reproductive potential if varicocele correction is performed for male infertility, given that the mean time to natural pregnancy after varicocele surgery is 8 months (16). In general, it is not advisable to repair varicoceles in with known genetic infertility, as the chance of improving either semen quality or natural pregnancy rates is low (17).

In addition to clinical arguments that suggest varicocele repair benefits infertility, economic analyses also support this concept. Cost-benefit and decision modeling arguments have shown that varicocelectomy is more cost effective than ART procedures $(11,12)$. More recently, it has been demonstrated in "shift of care" analyses that as many as $50 \%$ of couples who would only be candidates for ART procedures due to low semen quality can be "rescued" from such procedures and conceive naturally or with IUI after varicocelectomy (16).

\section{Reproductive Tract Obstruction}

Idiopathic epididymal obstruction is a relatively uncommon condition found in otherwise healthy men. Although many cases may result from prior infection, there is recent evidence linking this condition to cystic fibrosis in that one-third of men may harbor CFTR gene mutations (18). Inguinal vasal obstruction is an iatrogenic sequel of the use of knitted polypropylene monofilament mesh prosthesis for inguinal herniorrhaphy. This "plug and patch" repair method is currently performed in $80 \%$ of inguinal hernia repairs. The postoperative inflammatory response to mesh can result in fibrosis, which may entrap and obstruct the inguinal vas deferens. The exact incidence of this problem is currently undefined (19). Young syndrome presents with a triad of chronic sinusitis, bronchiectasis, and obstructive azoospermia, with obstruction in the epididymis. The pathophysiology of the condition is unclear but may involve abnormal ciliary function or abnormal mucus quality (20). Lastly, obstruction due to vasectomy is very common in many countries. Reversal of vasectomy by either vasovasostomy or epididymovasostomy is usually more successful than when microsurgery performed to correct any of the other conditions listed above, and is highly dependent on the skill and technique of the surgeon.

Ejaculatory duct obstruction (EDO) is a unique form of blockage within the male reproductive tract. It presents with low volume azoospermia (complete obstruction) or low volume oligoasthenospermia (partial obstruction). Obstruction can be congenital and result from Müllerian duct (utricular) cysts, Wolffian duct (diverticular) cysts, or atresia, or can be acquired from seminal vesicle calculi or postsurgical or inflammatory scar tissue. Based on a suspicious semen analysis, transrectal ultrasound (TRUS) is performed to confirm the diagnosis. Recent literature suggests that: a) TRUS, as a static test, can overdiagnose EDO in up to $50 \%$ of cases as some "obstruction" may be functional and not physical, b) the addition of "dynamic" imaging prior to surgical treatment, such as ejaculatory duct chromotubation or vasography, can distinguish true obstruction from functional obstruction, and c) partial obstruction is more difficult to diagnose than complete obstruction as TRUS findings are less specific for partial obstruction (21). Overall, a 20$30 \%$ pregnancy rate can be expected from endoscopic treatment of EDO, and $70-80 \%$ of men will achieve a significant improvement in semen quality. Complications occur in $10 \%$ of cases and include hematuria, watery ejaculate and epididymitis.

\section{Uncorrectable Conditions in the Male}

\section{Chemotherapy and Radiotherapy}

Chemotherapy is designed to kill rapidly dividing malignant or diseased cells; an undesired outcome is the cytotoxic effect on normal tissues. Differentiating spermatogonia appear to be the germ 
cells most sensitive to chemotherapy. Alkylating agents such as cyclophosphamide, chlorambucil, and nitrogen mustard are the most toxic agents. A list of agents and their relative toxicity to the testis (from decreasing to increasing effect) is given in Table- 6 . The toxicity of chemotherapy varies widely according to dose and duration of treatment, type and stage of disease, age and health of the patient, and baseline testis function. Despite this toxicity, the mutagenic effects of chemotherapy do not appear to be significant enough to increase the chance of congenital defects or genetically linked diseases among naturally conceived offspring. However, patients should wait at least 6 months after chemotherapy ends before attempting to conceive. Men with continued azoospermia after treatment are very likely $(65 \%)$ to have testis sperm available for use with IVF and ICSI (22).

The effects of radiotherapy on sperm production are well described and derived mainly from a series of experiments performed on healthy prisoners in Oregon and Washington in the 1960s (23). From these studies (Level B evidence), a significant reduction in sperm count is observed at $15 \mathrm{cGy}$ exposure, and sperm counts are temporarily abolished at $50 \mathrm{cGy}$. Persistent azoospermia is induced at 400 cGy. Recovery from radiation exposure can take up to 1 year. Similar to chemotherapy, there does not appear to be an increase in congenital birth defects in naturally conceived offspring of irradiated men.

\section{Congenital or Acquired Obstruction}

Cystic fibrosis (CF) is the most common autosomal recessive genetic disorder in the United States. It is caused by CFTR mutations and is fatal. It is associated with fluid and electrolyte abnormalities (abnormal chloride-sweat test) and presents with chronic lung obstruction and infections, pancreatic insufficiency, and infertility. Interestingly, 99\% of men with CF have mesonephric duct abnormalities, including absent of atrophic vas deferens, seminal vesicles, and ejaculatory ducts. Spermatogenesis is usually normal. Another group of men without obvious signs of CF may also exhibit reproductive tract abnormalities that are unreconstructable (congenital absence of the vas deferens, CAVD). This is a form of $\mathrm{CF}$, since $80 \%$ of these patients will harbor a detectable CF mutation and $15 \%$ will have renal

Table 6 - Toxic effects of chemotherapy on the testis.

\begin{tabular}{lll}
\hline Agent & Recoverability & Full Recovery Time \\
\hline Cisplatin & Good & 2 years \\
Methotrexate & Good & 1 year \\
Cytosine arabinoside & Good & 1 year \\
6-Mercatopurine & Good & 1 year \\
Thioguanine & Good & 1 year \\
Vincristine & Good & 6 months \\
Prednisone & Good & 1 year \\
Androgens & Good & 1 year \\
Estrogens & Good & 1 year \\
Doxorubicin & Good & 2 years \\
Cisplatin, etoposide and bleomycin & Good & 2 years \\
Vinblastine, bleomycin and cisplatin & Good & 3 years \\
Cyclophosphamide, vincristine, procarbazine and prednisone & Moderate & 4 years \\
Doxorubicin, bleomycin, vinblastine and decarbazine & Moderate & 5 years \\
Cyclophosphamide & Poor & 5 years \\
Chlorambucil & Poor & 5 years \\
Procarbazine & Poor & 5 years \\
Nitrogen mustard, vincristine, procarbazine and prednisone & Very poor & \\
\hline
\end{tabular}


malformations, most commonly unilateral agenesis (24). In either case, sperm retrieval from the testis or epididymis is effective in most cases (25). Genetic counseling the testing in these patients is important to define the residual risk of $\mathrm{CF}$ disease transmission to offspring (26).

Adult polycystic kidney disease is an autosomal dominant disorder associated with numerous cysts of the kidney, liver, spleen, pancreas, epididymis, seminal vesicle, and testis. Disease onset usually occurs in the twenties or thirties with symptoms of abdominal pain, hypertension, and renal failure. Infertility with this disease is usually secondary to obstructing cysts in the epididymis or seminal vesicle. Functional blockages may result from nerve injury or medications that impair the contractility of seminal vesicle or vasal musculature. A classic example is nerve injury after retroperitoneal lymph node dissection for testis cancer, causing either retrograde ejaculation or complete anejaculation, depending on the degree of injury to postganglionic sympathetic fibers. Multiple sclerosis, spinal cord injury and diabetes are other conditions that result in disordered ejaculation. Some forms of anejaculation are treatable with rectal probe electroejaculation or penile vibratory stimulation along with intrauterine insemination; others are currently not correctable and require higher forms of assisted reproduction for fatherhood (27).

\section{Genetic Male Infertility}

Overall, $50 \%$ of male infertility is currently unexplained and it is likely that much of this is has a genetic basis. Over the last decade, a strong association has arisen between deletions in several regions of the $\mathrm{Y}$ chromosome and male infertility. They are termed the Azoospermia Factor regions $\mathrm{AZFa}, \mathrm{AZFb}, \mathrm{AZFc}$. The relative frequency of individual AZF deletions is illustrated in Figure-3 (7). Another group of men with oligospermia (2\%) or azoospermia (15\%) will harbor abnormalities in chromosomal number or constitution as assessed by a karyotype analysis. In general, affected men present with testis atrophy and elevated follicle stimulating hormone levels, similar to non-genetic infertility. In the presence of genetic infertility, there is no evidence that classical medical or surgical treatments for male infertility will be successful (17). Instead, affected patients should proceed to assisted reproduction with either ejaculated or surgically retrieved sperm.

One large obstacle to biological family building in men with genetic infertility and azoospermia is the fact that only $50-60 \%$ of men with this condition will harbor usable sperm in the testis form IVF-ICSI. This state of affairs is also true in men with nonobstructive azoospermia in which a genetic etiology is not formally defined, but only suspected. Unfortunately, clinical features of testicular size, history of ejaculated sperm, serum FSH level, or biopsy histology do not accurately predict whether or not sperm will be recovered on exploration. Because of this, strategies have been developed to more accurately determine which men with failing testes are candidates for IVF-ICSI, and surgical techniques have been refined to minimize the invasiveness of sperm harvest procedures. One strategy involves taking as many biopsy samples as needed either before IVF-ICSI (and freezing the sperm for later thaw and use), or at the time of ICSI (28). Microdissection TESE uses microsurgical exploration of the widely opened testis to search for pockets of sperm (29). Finally, diagnostic testis fine needle aspiration (FNA) "mapping" seeks to determine patient candidacy for future sperm testis retrieval (30).

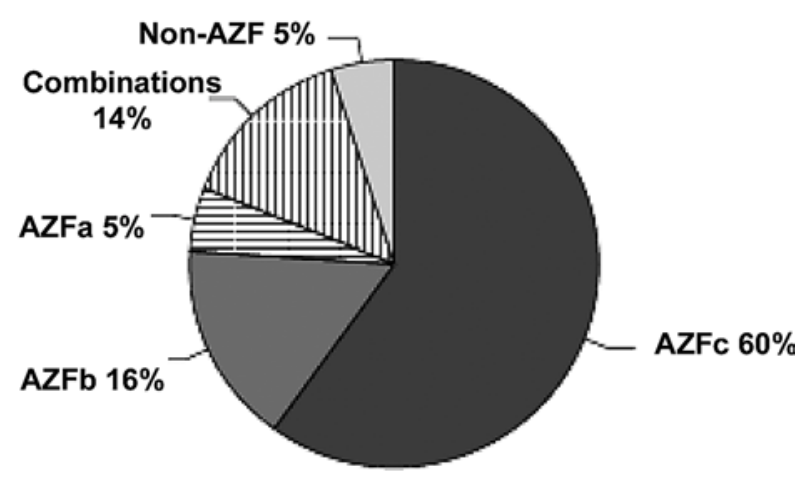

Figure 3 - The relative frequency of specific AZF deletions in men $(n=5000)$ with Yq microdeletions. 
Subsequently, at ICSI, needles or biopsies are "directed" to testis locations informed by the map. Armed with the knowledge of sperm presence from prior "mapping" we have found that the need for testis microdissection during sperm retrieval can be reduced to roughly $20 \%$ of cases, with the vast majority requiring less invasive methods to retrieve sperm, including TESA (testis sperm aspiration by needle) or TESE (testis sperm extraction by small biopsy). A review of 8 single institution studies using either microdissection or FNA mapping suggests that about $50 \%$ of nonobstructive azoospermic men with have sperm found by either technique.

Over the last decade, several interesting observations have been made regarding genetic infertility and the potential to father children. It appears that the presence or absence of sperm (ejaculated or retrieved) in men with Y chromosome microdeletions varies depending on the specific deletion. In contrast to partial and complete AZFc deletion patients, in whom sperm can be found on semen analysis or in the testis $70 \%$ of the time, the chance of finding sperm in men with complete AZFa or $\mathrm{AZFb}$ deletions is unlikely (31). However, since fewer than 100 patients with isolated AZFa or AZFb deletions have been reported, more patients are needed to confirm this statement. In addition, from four studies that have addressed the IVF-ICSI outcomes in men with Y deletions (all AZFc deleted patients), it appears that fertilization rates, embryo quality and pregnancy rates approximate non Ydeleted contemporary controls. Furthermore, it has been shown that men with AZF deletions who conceive with IVF-ICSI will pass on the Yq deletion to male offspring despite being somatically healthy. Lastly, there has been an observation that there may be a time-dependent decline in sperm production in Yq deleted patients, which leads some to suggest that affected patients should consider cryopreservation of ejaculated sperm in early adulthood (32).

\section{CONFLICT OF INTEREST}

None declared.

\section{REFERENCES}

1. Honig SC, Lipshultz LI, Jarow JP: Significant medical pathology uncovered by a comprehensive male infertility evaluation. Fert Steril. 1994; 62: 1028-34.

2. WHO Laboratory Manual for the Examination of Human Semen and Semen-Cervical Mucus Interaction, Fourth edition. Cambridge, Cambridge University Press. 1999; pp. 23-25.

3. Guzick DS, Overstreet JW, Factor-Litvak P, Brazil CK, Nakajima ST, Coutifaris C, et al.: Sperm morphology, motility, and concentration in fertile and infertile men. N Engl J Med. 2001; 345: 1388-93.

4. Misell LM, Holochwost D, Boban D, Santi N, Shefi $\mathrm{S}$, Hellerstein MK, et al.: A stable isotope-mass spectrometric method for measuring human spermatogenesis kinetics in vivo. J Urol. 2006; 175: 242-6; discussion 246.

5. Kruger TF, Acosta AA, Simmons KF, Swanson RJ, Matta JF, Oehninger S: Predictive value of abnormal sperm morphology in in vitro fertilization. Fertil Steril. 1988; 49: 112-7.

6. Sigman M, Jarow JP: Endocrine evaluation of infertile men. Urology. 1997; 50: 659-64.

7. Foresta C, Moro E, Ferlin A: Y chromosome microdeletions and alterations of spermatogenesis. Endocr Rev. 2001; 22: 226-39.

8. Turek PJ: Practical approach to the diagnosis and management of male infertility. Nature Clin Pract Urol. 2005; 2: 1-13.

9. Turek PJ, Lipshultz LI: Immunologic infertility. Urol Clin North Am. 1994; 21: 447-68.

10. Evenson DP, Jost LK, Marshall D, Zinaman MJ, Clegg E, Purvis K, et al.: Utility of the sperm chromatin structure assay as a diagnostic and prognostic tool in the human fertility clinic. Hum Reprod. 1999; 14: 103949.

11. Meng MV, Greene KL, Turek PJ: Surgery or assisted reproduction? A decision analysis of treatment costs in male infertility. J Urol. 2005; 174: 1926-31; discussion 1931.

12. Schlegel PN: Is assisted reproduction the optimal treatment for varicocele-associated male infertility? A cost-effectiveness analysis. Urology. 1997; 49: 83-90.

13. Wilcox AJ, Weinberg CR, Baird DD: Timing of sexual intercourse in relation to ovulation. Effects on the probability of conception, survival of the pregnancy, and sex of the baby. N Engl J Med. 1995; 333: 151721. 
14. Evers JL, Collins JA: Assessment of efficacy of varicocele repair for male subfertility: a systematic review. Lancet. 2003; 361: 1849-52.

15. No authors listed: The influence of varicocele on parameters of fertility in a large group of men presenting to infertility clinics. World Health Organization. Fertil Steril. 1992; 57: 1289-93.

16. Cayan S, Erdemir F, Ozbey I, Turek PJ, Kadioglu A, Tellaloglu S: Can varicocelectomy significantly change the way couples use assisted reproductive technologies? J Urol. 2002; 167: 1749-52.

17. Cayan S, Lee D, Black LD, Reijo Pera RA, Turek PJ: Response to varicocelectomy in oligospermic men with and without defined genetic infertility. Urology. 2001; 57: 530-5.

18. Jarvi K, Zielenski J, Wilschanski M, Durie P, Buckspan M, Tullis E, et al.: Cystic fibrosis transmembrane conductance regulator and obstructive azoospermia. Lancet. 1995; 345: 1578.

19. Shin D, Lipshultz LI, Goldstein M, Barme GA, Fuchs EF, Nagler HM, et al.: Herniorrhaphy with polypropylene mesh causing inguinal vasal obstruction: a preventable cause of obstructive azoospermia. Ann Surg. 2005; 241: 553-8.

20. Handelsman DJ, Conway AJ, Boylan LM, Turtle JR: Young's syndrome. Obstructive azoospermia and chronic sinopulmonary infections. N Engl J Med. 1984; 310: 3-9.

21. Purohit RS, Wu DS, Shinohara K, Turek PJ: A prospective comparison of 3 diagnostic methods to evaluate ejaculatory duct obstruction. J Urol. 2004; 171: 232-5; discussion 235-6.

22. Damani MN, Master V, Meng MV, Burgess C, Turek P, Oates RD, et al.: Postchemotherapy ejaculatory azoospermia: fatherhood with sperm from testis tissue with intracytoplasmic sperm injection. J Clin Oncol 2002; 20: 930-6.

\section{Correspondence address:}

Dr. Paul J. Turek

Endowed Chair in Urologic Education

Department of Urology - UCSF

1600 Divisadero St, Rm. A633

San Francisco, CA 94143-1695

Fax: + 1415 885-7443

E-mail:pturek@urology.ucsf.edu
23. Clifton DK, Bremner WJ: The effect of testicular xirradiation on spermatogenesis in man. A comparison with the mouse. J Androl. 1983; 4: 387-92.

24. Chillon M, Casals T, Mercier B, Bassas L, Lissens W, Silber S, et al.: Mutations in the cystic fibrosis gene in patients with congenital absence of the vas deferens. N Engl J Med. 1995; 332: 1475-80.

25. Nudell DM, Conaghan J, Pedersen RA, Givens CR, Schriock ED, Turek PJ: The mini-micro-epididymal sperm aspiration for sperm retrieval: a study of urological outcomes. Hum Reprod. 1998; 13: 1260-5.

26. Danziger KL, Black LD, Keiles SB, Kammesheidt A, Turek PJ: Improved detection of cystic fibrosis mutations in infertility patients with DNA sequence analysis. Hum Reprod. 2004; 19: 540-6.

27. Master VA, Turek PJ: Ejaculatory physiology and dysfunction. Urol Clin North Am. 2001; 28: 363-75.

28. Mulhall JP, Burgess CM, Cunningham D, Carson R, Harris D, Oates RD: Presence of mature sperm in testicular parenchyma of men with nonobstructive azoospermia: prevalence and predictive factors. Urology. 1997; 49: 91-5; discussion 95-6.

29. Schlegel PN: Testicular sperm extraction: microdissection improves sperm yield with minimal tissue excision. Hum Reprod. 1999; 14: 131-5.

30. Turek PJ, Ljung BM, Cha I, Conaghan J: Diagnostic findings from testis fine needle aspiration mapping in obstructed and nonobstructed azoospermic men. J Urol. 2000; 163: 1709-16.

31. Hopps CV, Mielnik A, Goldstein M, Palermo GD, Rosenwaks Z, Schlegel PN: Detection of sperm in men with Y chromosome microdeletions of the AZFa, AZFb and AZFc regions. Hum Reprod. 2003; 18: 1660-5.

32. Krausz C, Bussani-Mastellone C, Granchi S, McElreavey K, Scarselli G, Forti G: Screening for microdeletions of $\mathrm{Y}$ chromosome genes in patients undergoing intracytoplasmic sperm injection. Hum Reprod. 1999; 14: 1717-21.
Accepted:

February 3, 2006 